\title{
THE
}

UNIVERSITY

University of Rhode Island

OF RHODE ISLAND

DigitalCommons@URI

$11-22-2003$

\section{Energy Estimators for Random Series Path-Integral Methods}

Cristian Predescu

Dubravko Sabo

J. D. Doll

David L. Freeman

University of Rhode Island, dfreeman@uri.edu

Follow this and additional works at: https://digitalcommons.uri.edu/chm_facpubs

Terms of Use

All rights reserved under copyright.

\section{Citation/Publisher Attribution}

Predescu, C., Sabo, D., Doll, J. D., \& Freeman, D. L. (2003). Energy Estimators for Random Series PathIntegral Methods. Journal of Chemical Physics, 119(20), 10475-10488. doi: 10.1063/1.1619372

Available at: http://dx.doi.org/10.1063/1.1619372

This Article is brought to you for free and open access by the Chemistry at DigitalCommons@URI. It has been accepted for inclusion in Chemistry Faculty Publications by an authorized administrator of DigitalCommons@URI. For more information, please contact digitalcommons-group@uri.edu. 


\title{
ARTICLES
}

\section{Energy estimators for random series path-integral methods}

\author{
Cristian Predescu, ${ }^{\text {a) }}$ Dubravko Sabo, and J. D. Doll \\ Department of Chemistry, Brown University, Providence, Rhode Island 02912 \\ David L. Freeman \\ Department of Chemistry, University of Rhode Island, Kingston, Rhode Island 02881
}

(Received 28 May 2003; accepted 25 August 2003)

\begin{abstract}
We perform a thorough analysis on the choice of estimators for random series path integral methods. In particular, we show that both the thermodynamic (T-method) and the direct (H-method) energy estimators have finite variances and are straightforward to implement. It is demonstrated that the agreement between the T-method and the $\mathrm{H}$-method estimators provides an important consistency check on the quality of the path integral simulations. We illustrate the behavior of the various estimators by computing the total, kinetic, and potential energies of a molecular hydrogen cluster using three different path integral techniques. Statistical tests are employed to validate the sampling strategy adopted as well as to measure the performance of the parallel random number generator utilized in the Monte Carlo simulation. Some issues raised by previous simulations of the hydrogen cluster are clarified. (C) 2003 American Institute of Physics. [DOI: 10.1063/1.1619372]
\end{abstract}

\section{INTRODUCTION}

Numerical path integral methods have proved to be highly useful tools in the analysis of finite temperature, many-body quantum systems. ${ }^{1}$ A central theme in such studies is the conscious use of dimensionality, both in the reformulation of the original problem and in the subsequent numerical simulations.

As the scale of the problems under study continues to grow, it becomes increasingly important that the formal properties of the numerical methods that are utilized be properly characterized. Recently, Predescu and co-workers ${ }^{2-4}$ have presented a number of results concerning the convergence properties of random series-based path integral techniques. Important in their own right, these formal properties have also led to the development of a new class of path integral methods, the so-called reweighted techniques. ${ }^{4} \mathrm{Re}-$ weighted approaches accelerate the convergence of "primitive" series methods by including the effects of "higherorder" path variables in a simple, approximate fashion. Reweighted methods achieve the convergence rate of related partial averaging approaches ${ }^{5}$ without requiring the construction of the Gaussian transform of the underlying potential energy function.

Previous work on the reweighted method has focused principally on the construction of the quantum-mechanical density matrix. ${ }^{4,6}$ In the present work, we wish to examine estimators for various coordinate-diagonal and off-diagonal properties. While the present discussion is focused principally on reweighted methods, the results obtained are broadly applicable to more general random series approaches.

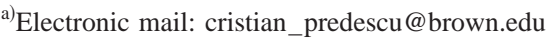

In Sec. II of the present article, we examine the thermodynamic (T-method) and direct (H-method) estimators for the total energy. In order to avoid any confusion with earlier estimators, we mention that in the present article by T-method and H-method estimators we understand the respective energy estimators introduced by Predescu and Doll in Ref. 2. Thus, the T-method estimator we employ does not have the variance difficulties associated with the Barker estimator for large numbers of path variables. ${ }^{7}$ As the lowtemperature simulation presented in the second part of the article demonstrates, the present T-method estimator does not exhibit any of the difficulties sometimes associated with the virial estimator for low-temperature systems or for strongly correlated Monte Carlo sampling techniques. ${ }^{8-12}$ The T-method estimator is closely related and similar in form to the centroid virial estimator. ${ }^{13,14}$ We expect the two estimators to have similar behavior with the nature of the quantum system, the temperature, and the Monte Carlo sampling method. However, an important difference between the two estimators is the fact that the T-method estimator is a veritable thermodynamic estimator, in the sense that it is obtained by temperature differentiation of the quantum partition function. This observation is important because the temperature differentiation can be implemented numerically by a finite-difference scheme and, in principle, may lead to numerically stable algorithms that do not require derivatives of the potential. For large dimensional systems or systems described by complicated potentials, we expect such algorithms to be significantly faster than those based on explicit analytical formulas. The relative merits of such algorithms will be examined in future work.

In Sec. III, we examine the application of the reweighted methods to a model problem, that of simulating the thermodynamic properties of the $\left(\mathrm{H}_{2}\right)_{22}$ molecular cluster. In Sec. 
IV, we summarize our present findings and clarify a number of issues raised in previous studies of this molecular hydrogen system. ${ }^{15,16}$

\section{ENERGY ESTIMATORS}

In this section, we consider a one-dimensional quantum canonical system characterized by inverse temperature $\beta$ $=1 /\left(k_{B} T\right)$ and set forward the task of computing its average energy by Monte Carlo integration methods developed around several reweighted techniques. ${ }^{4,6}$ The physical system is made up of a particle of mass $m_{0}$ moving in the potential $V(x)$. We discuss the numerical implementation and the computational merits of both the T-method and H-method estimators. Any time the multidimensional extension is not obvious, we present the explicit formulas of the respective estimators.

We begin by presenting the general form of the path integral methods we employ in this paper. We remind the reader that in terms of a standard Brownian motion $\left\{B_{u}, u\right.$ $\geqslant 0\}$, the Feynman-Kaç formula has the expression ${ }^{17}$

$$
\begin{aligned}
\rho\left(x, x^{\prime} ; \beta\right)= & P\left[\sigma B_{1}=x^{\prime} \mid \sigma B_{0}=x\right] \\
& \times \mathbb{E}\left[e^{-\beta \int_{0}^{1} V\left(\sigma B_{u}\right) d u} \mid \sigma B_{1}=x^{\prime}, \sigma B_{0}=x\right],
\end{aligned}
$$

where $\sigma=\left(\hbar^{2} \beta / m_{0}\right)^{1 / 2}$. In this paper, we shall use the symbol $\mathbb{E}$ to denote the expected value (average value) of a certain random variable against the underlying probability measure of the Brownian motion $B_{u}$. It is straightforward to see that the first factor of the product in Eq. (1) (which represents the conditional probability density that the rescaled Brownian motion $\sigma B_{u}$ reaches the point $x^{\prime}$ provided that it starts at the point $x$ ) is the density matrix of a free particle of mass $m_{0}$

$$
P\left[\sigma B_{1}=x^{\prime} \mid \sigma B_{0}=x\right]=\rho_{f p}\left(x, x^{\prime} ; \beta\right) .
$$

Moreover, rather than using the conditional expectation appearing in the second factor of Eq. (1), one usually employs a stochastic process $\left\{B_{u}^{0} ; 0 \leqslant u \leqslant 1\right\}$, called a standard Brownian bridge, ${ }^{17,18}$ which is defined as a standard Brownian motion conditioned on the end points such that $B_{0}^{0}=0$ and $B_{1}^{0}=0$. In terms of the newly defined process, the Feynman-Kaç formula reads

$$
\frac{\rho\left(x, x^{\prime} ; \beta\right)}{\rho_{f p}\left(x, x^{\prime} ; \beta\right)}=\mathbb{E} \exp \left\{-\beta \int_{0}^{1} V\left[x_{r}(u)+\sigma B_{u}^{0}\right] d u\right\},
$$

where $x_{r}(u)=x+\left(x^{\prime}-x\right) u$ is a straight line connecting the points $x$ and $x^{\prime}$ and is called the reference path.

As discussed in Ref. 2, one of the most general constructions of the standard Brownian bridge is given by the ItoNisio theorem. ${ }^{19}$ Let $\left\{\lambda_{k}(\tau)\right\}_{k \geqslant 1}$ be a system of functions on the interval $[0,1]$, which together with the constant function $\lambda_{0}(\tau)=1$, make up an orthonormal basis in $L^{2}[0,1]$. Let

$$
\Lambda_{k}(t)=\int_{0}^{t} \lambda_{k}(u) d u .
$$

If $\Omega$ is the space of infinite sequences $\bar{a} \equiv\left(a_{1}, a_{2}, \ldots\right)$ and

$$
d P[\bar{a}]=\prod_{k=1}^{\infty} d \mu\left(a_{k}\right),
$$

is the probability measure on $\Omega$ such that the coordinate maps $\bar{a} \rightarrow a_{k}$ are independent identically distributed (i.i.d.) variables with distribution probability

$$
d \mu\left(a_{i}\right)=\frac{1}{\sqrt{2 \pi}} e^{-a_{i}^{2} / 2} d a_{i},
$$

then

$$
B_{u}^{0}(\bar{a})=\sum_{k=1}^{d} a_{k} \Lambda_{k}(u), \quad 0 \leqslant u \leqslant 1
$$

i.e., the right-hand side random series is equal in distribution to a standard Brownian bridge. The notation $B_{u}^{0}(\bar{a})$ in (4) is then appropriate and allows us to interpret the Brownian bridge as a collection of random functions of argument $\bar{a}$, indexed by $u$.

Using the Ito-Nisio representation of the Brownian bridge, the Feynman-Kaç formula takes the form

$$
\begin{aligned}
\frac{\rho\left(x, x^{\prime} ; \beta\right)}{\rho_{f p}\left(x, x^{\prime} ; \beta\right)}= & \int_{\Omega} d P[\bar{a}] \exp \left\{-\beta \int_{0}^{1} V\left[x_{r}(u)\right.\right. \\
& \left.\left.+\sigma \sum_{k=1}^{\infty} a_{k} \Lambda_{k}(u)\right] d u\right\} .
\end{aligned}
$$

For a multidimensional system, the Feynman-Kaç formula is obtained by employing an independent random series for each additional degree of freedom.

A reweighted method constructed from the random series $\sum_{k=1}^{\infty} a_{k} \Lambda_{k}(u)$ is any sequence of approximations to the density matrix of the form ${ }^{4}$

$$
\begin{aligned}
\frac{\rho_{n}^{\mathrm{RW}}\left(x, x^{\prime} ; \beta\right)}{\rho_{f p}\left(x, x^{\prime} ; \beta\right)}= & \int_{\mathrm{R}} d \mu\left(a_{1}\right) \ldots \int_{\mathrm{R}} d \mu\left(a_{q n+p}\right) \\
& \times \exp \left\{-\beta \int_{0}^{1} V\left[x_{r}(u)\right.\right. \\
& \left.\left.+\sigma \sum_{k=1}^{q n+p} a_{k} \tilde{\Lambda}_{n, k}(u)\right] d u\right\},
\end{aligned}
$$

where $q$ and $p$ are some fixed integers, where

$$
\tilde{\Lambda}_{n, k}(u)=\Lambda_{k}(u) \text { if } 1 \leqslant k \leqslant n,
$$

and where

$$
\sum_{k=n+1}^{q n+p} \tilde{\Lambda}_{n, k}(u)^{2}=\sum_{k=n+1}^{\infty} \Lambda_{k}(u)^{2} .
$$

In Eq. (6), $n$ indexes the sequence of reweighted approximations $\rho_{n}^{\mathrm{RW}}\left(x, x^{\prime} ; \beta\right)$, sequence that converges to the density matrix $\rho\left(x, x^{\prime} ; \beta\right)$ in the limit $n \rightarrow \infty$. Remark that the approximation of index $n$ actually utilizes $q n+p$ variables for path parameterization. In the construction of a certain path, the first $n$ functions $\widetilde{\Lambda}_{n, k}(u)$ coincide with the ones for the corresponding series representation, as shown by Eq. (7). A number of $(q-1) n+p$ additional functions are constructed 
so that to maximize the order of convergence of the reweighted approximation. Notice that if the resulting approximation has a convergence of order $\alpha$ as measured against $n$, then it has the same order of convergence when measured against the total number of variables $q n+p$, though the convergence constant is $q^{\alpha}$ times larger. This explains why the number of additional functions is chosen to scale linearly with $n$. For additional information, the reader is advised to consult Ref. 4.

It is convenient to introduce the additional quantities $X_{n}\left(x, x^{\prime}, \bar{a} ; \beta\right)$ and $X_{\infty}\left(x, x^{\prime}, \bar{a} ; \beta\right)$, which are defined by the expressions

$$
\begin{aligned}
X_{n}\left(x, x^{\prime}, \bar{a} ; \beta\right)= & \rho_{f p}\left(x, x^{\prime} ; \beta\right) \exp \left\{-\beta \int_{0}^{1} V\left[x_{r}(u)\right.\right. \\
& \left.\left.+\sigma \sum_{k=1}^{q n+p} a_{k} \tilde{\Lambda}_{n, k}(u)\right] d u\right\}
\end{aligned}
$$

and

$$
\begin{aligned}
X_{\infty}\left(x, x^{\prime}, \bar{a} ; \beta\right)= & \rho_{f p}\left(x, x^{\prime} ; \beta\right) \exp \left\{-\beta \int_{0}^{1} V\left[x_{r}(u)\right.\right. \\
& \left.\left.+\sigma \sum_{k=1}^{\infty} a_{k} \Lambda_{k}(u)\right] d u\right\},
\end{aligned}
$$

respectively. With the new notation, Eq. (6) becomes

$$
\rho_{n}^{\mathrm{RW}}\left(x, x^{\prime} ; \beta\right)=\int_{\Omega} d P[\bar{a}] X_{n}\left(x, x^{\prime}, \bar{a} ; \beta\right),
$$

while the Feynman-Kaç formula reads

$$
\rho\left(x, x^{\prime} ; \beta\right)=\int_{\Omega} d P[\bar{a}] X_{\infty}\left(x, x^{\prime}, \bar{a} ; \beta\right) .
$$

The analytical expressions of the functions $\tilde{\Lambda}_{n, k}(u)$ depend on the nature of the reweighted techniques and are generally chosen to maximize the asymptotic convergence of the respective reweighted techniques. ${ }^{4}$ To a large extent, the specific form of these functions is not important for the present development, but the reader is advised to consult Refs. 4 and 6 for quadrature techniques and additional clarifications.

The remainder of the present section is split into two parts. First, we discuss the problem of computing the ensemble averages of operators diagonal in coordinate representation. In particular, this resolves the problem of computing the average potential energy. Second, we consider the problem of evaluating the total energies (hence, also the kinetic energies) by means of the T-method and H-method estimators.

\section{A. Operators diagonal in the coordinate representation}

By definition, the ensemble average of an operator $\hat{O}$ diagonal in the coordinate representation is

$$
\langle O\rangle_{\beta}=\frac{\int_{\mathbb{R}} \rho(x ; \beta) O(x) d x}{\int_{\mathbb{R}} \rho(x ; \beta) d x} .
$$

The quantity $\rho(x ; \beta)=\rho(x, x ; \beta)$ is the diagonal density matrix. By convention, we drop the second variable of the pair $\left(x, x^{\prime}\right)$ any time $x=x^{\prime}$. For instance, we use $X_{n}(x, \bar{a} ; \beta)$ instead of $X_{n}(x, x, \bar{a} ; \beta)$. By means of Eq. (12), the average above can be recast as

$$
\langle O\rangle_{\beta}=\frac{\int_{\mathbb{R}} d x \int_{\Omega} d P[\bar{a}] X_{\infty}(x, \bar{a} ; \beta) O(x)}{\int_{\mathbb{R}} d x \int_{\Omega} d P[\bar{a}] X_{\infty}(x, \bar{a} ; \beta)} .
$$

This average can be recovered as the limit $n \rightarrow \infty$ of the sequence

$$
\langle O\rangle_{\beta, n}^{\mathrm{pt}}=\frac{\int_{\mathbb{R}} d x \int_{\Omega} d P[\bar{a}] X_{n}(x, \bar{a} ; \beta) O(x)}{\int_{\mathbb{R}} d x \int_{\Omega} d P[\bar{a}] X_{n}(x, \bar{a} ; \beta)},
$$

the terms of which are to be evaluated by Monte Carlo integration. The estimating function $O(x)$ appearing in the above formula is called the point estimating function of the operator $\hat{O}$.

An alternative to the point estimating function is the socalled path estimating function, the derivation of which is presented shortly. As demonstrated in the Appendix, the function $O(x)$ appearing in Eq. (14) can be replaced by $O\left[x+\sigma B_{u}^{0}(\bar{a})\right]$, without changing the value of the average $\langle O\rangle_{\beta}$. That is, the equality

$$
\langle O\rangle_{\beta}=\frac{\int_{\mathbb{R}} d x \int_{\Omega} d P[\bar{a}] X_{\infty}(x, \bar{a} ; \beta) O\left[x+\sigma B_{u}^{0}(\bar{a})\right]}{\int_{\mathbb{R}} d x \int_{\Omega} d P[\bar{a}] X_{\infty}(x, \bar{a} ; \beta)},
$$

is valid for all $0 \leqslant u \leqslant 1$. Averaging over the variable $u$, one obtains

$$
\langle O\rangle_{\beta}=\frac{\int_{\mathbb{R}} d x \int_{\Omega} d P[\bar{a}] X_{\infty}(x, \bar{a} ; \beta) \int_{0}^{1} O\left[x+\sigma B_{u}^{0}(\bar{a})\right] d u}{\int_{\mathbb{R}} d x \int_{\Omega} d P[\bar{a}] X_{\infty}(x, \bar{a} ; \beta)} .
$$

Equation (16) shows that the ensemble average of the operator $\hat{O}$ can also be recovered as the limit $n \rightarrow \infty$ of the sequence

$\langle O\rangle_{\beta, n}^{\text {pth }}$

$$
=\frac{\int_{\mathrm{R}} d x \int_{\Omega} d P[\bar{a}] X_{n}(x, \bar{a} ; \beta) \int_{0}^{1} O\left[x+\sigma \widetilde{B}_{u, n}^{0}(\bar{a})\right] d u}{\int_{\mathbb{R}} d x \int_{\Omega} d P[\bar{a}] X_{n}(x, \bar{a} ; \beta)},
$$

where we have set

$$
\widetilde{B}_{u, n}^{0}(\bar{a})=\sum_{k=1}^{q n+p} a_{k} \tilde{\Lambda}_{n, k}(u),
$$

for convenience of notation.

In the remainder of the present subsection, we discuss the relative merits of the point and path estimators. We first consider which of $\langle O\rangle_{\beta, n}^{\mathrm{pt}}$ and $\langle O\rangle_{\beta, n}^{\mathrm{pth}}$ is closer to $\langle O\rangle_{\beta}$ for a given $n$ assuming the averages given in Eqs. (15) and (17) are computed exactly. Let us notice that Eq. (15) can be put in the form

$$
\langle O\rangle_{\beta, n}^{\mathrm{pt}}=\frac{\int_{\mathrm{R}} d x \rho_{n}^{\mathrm{RW}}(x ; \beta) O(x)}{\int_{\mathbb{R}} d x \rho_{n}^{\mathrm{RW}}(x ; \beta)} .
$$

The probability distribution 


$$
\frac{\rho_{n}^{\mathrm{RW}}(x ; \beta) d x}{\int_{\mathrm{R}} \rho_{n}^{\mathrm{RW}}(x ; \beta) d x},
$$

represents the marginal distribution of the variable $x$ regarded as a random variable on the space $R \times \Omega$, which is endowed with the probability measure

$$
\frac{X_{n}(x, \bar{a} ; \beta) d x d P[\bar{a}]}{\int_{\mathbb{R}} d x \int_{\Omega} d P[\bar{a}] X_{n}(x, \bar{a} ; \beta)} .
$$

The reweighted techniques are designed so that the distribution given by Eq. (18) is as close as possible to the quantum statistical one, which is given by the expression

$$
\frac{\rho(x ; \beta) d x}{\int_{\mathbb{R}} \rho(x ; \beta) d x} .
$$

In designing the reweighted techniques, one seeks to optimize the rate of convergence of the sequence $\rho_{n}^{\mathrm{RW}}\left(x, x^{\prime} ; \beta\right)$ $\rightarrow \rho\left(x, x^{\prime} ; \beta\right)$ for all $x$ and $x^{\prime} .{ }^{4}$

For arbitrary $u$, the marginal distribution of $x$ $+\sigma B_{u, n}^{0}(\bar{a})$ is usually different from the one given by Eq. (18) and is not optimized. With few notable exceptions to be analyzed below, the points $x+\sigma B_{u, n}^{0}(\bar{a})$ for different $u$ are not equivalent, and their probability distribution may differ significantly from the quantum statistical one. (However, as shown in the Appendix, they become equivalent in the limit $n \rightarrow \infty$.) Therefore, especially for those reweighted techniques having fast asymptotic convergence, we expect the point estimator to be more rapidly convergent with $n$ than the path estimator.

An additional issue appearing in Monte Carlo computations is the variance of the two estimating functions $O(x)$ and $\int_{0}^{1} O\left[x+\sigma B_{u, n}^{0}(\bar{a})\right] d u$. In the limit $n \rightarrow \infty$, the variance of the point estimating function converges to

$$
\begin{aligned}
& \frac{\int_{\mathbb{R}} d x \int_{\Omega} d P[\bar{a}] X_{\infty}(x, \bar{a} ; \beta) O(x)^{2}}{\int_{\mathrm{R}} d x \int_{\Omega} d P[\bar{a}] X_{\infty}(x, \bar{a} ; \beta)}-\langle O\rangle_{\beta}^{2} \\
& =\frac{\int_{\mathbb{R}} d x \int_{\Omega} d P[\bar{a}] X_{\infty}(x, \bar{a} ; \beta) \int_{0}^{1} O\left[x+\sigma B_{u}^{0}(\bar{a})\right]^{2} d u}{\int_{\mathrm{R}} d x \int_{\Omega} d P[\bar{a}] X_{\infty}(x, \bar{a} ; \beta)} \\
& \quad-\langle O\rangle_{\beta}^{2},
\end{aligned}
$$

while the variance of the path estimating function converges to

$$
\begin{gathered}
\frac{\int_{\mathbb{R}} d x \int_{\Omega} d P[\bar{a}] X_{\infty}(x, \bar{a} ; \beta)\left\{\int_{0}^{1} O\left[x+\sigma B_{u}^{0}(\bar{a})\right] d u\right\}^{2}}{\int_{\mathbb{R}} d x \int_{\Omega} d P[\bar{a}] X_{\infty}(x, \bar{a} ; \beta)} \\
-\langle O\rangle_{\beta}^{2} .
\end{gathered}
$$

The Cauchy-Schwartz inequality implies

$$
\left\{\int_{0}^{1} O\left[x+\sigma B_{u}^{0}(\bar{a})\right] d u\right\}^{2} \leqslant \int_{0}^{1} O\left[x+\sigma B_{u}^{0}(\bar{a})\right]^{2} d u,
$$

and shows that the variance of the path estimating function is always smaller than that of the point estimating function. The actual decrease in the variance is not always significant because the points $x+\sigma B_{u}^{0}(\bar{a})$ for different $u$ are strongly correlated. Depending on the nature of the function $O(x)$, the variance decrease may not compensate the effort required to compute the average $\int_{0}^{1} O\left[x+\sigma \widetilde{B}_{u, n}^{0}(\bar{a})\right] d u$. However, if the function $O(x)$ is the potential $V(x)$, then the smaller variance of the path estimator is a desirable feature because the path average $\int_{0}^{1} V\left[x+\sigma \widetilde{B}_{u, n}^{0}(\bar{a})\right] d u$, which also enters the expression of $X_{n}(x, \bar{a} ; \beta)$, is computed anyway.

To summarize the findings of the present subsection, the point estimator provides a more accurate value but has a larger variance than the path estimator. We next ask if there are any methods for which one may construct an estimator providing the same values as the point estimator but having the variance of the path estimator. More precisely, we seek methods for which there is a division $0=u_{0} \leqslant u_{1} \leqslant \ldots \leqslant u_{q_{n}}$ $\leqslant u_{q_{n}+1}=1$ such that the mesh $\max _{0 \leqslant i \leqslant q_{n}}\left|u_{i+1}-u_{i}\right|$ converges to zero as $n \rightarrow \infty$ and such that the points $\{x$ $\left.+\sigma \widetilde{B}_{u_{i}, n}^{0}(\bar{a}) ; 0 \leqslant i \leqslant q_{n}+1\right\}$ have the same marginal distribution as $x$. For such methods, the expected value of the estimating function

$$
\sum_{i=0}^{q_{n}} O\left[x+\sigma \widetilde{B}_{u_{i}, n}^{0}(\bar{a})\right]\left(u_{i+1}-u_{i}\right),
$$

under the probability distribution given by Eq. (19) is an estimator satisfying the criteria outlined in this paragraph.

There are two methods we employ in the present paper for which such an estimator exists. The first one, is the trapezoidal Trotter discrete path integral method (TT-DPI) obtained by the Trotter composition

$$
\begin{aligned}
\rho_{n}^{\mathrm{TT}}\left(x, x^{\prime} ; \beta\right)= & \int_{\mathbb{R}} d x_{1} \ldots \int_{\mathbb{R}} d x_{n} \rho_{0}\left(x, x_{1} ; \frac{\beta}{n+1}\right) \\
& \ldots \rho_{0}\left(x_{n}, x^{\prime} ; \frac{\beta}{n+1}\right),
\end{aligned}
$$

of the short-time approximation

$$
\rho_{0}^{\mathrm{TT}}\left(x, x^{\prime} ; \beta\right)=\rho_{f p}\left(x, x^{\prime} ; \beta\right) \exp \left[-\beta \frac{V(x)+V\left(x^{\prime}\right)}{2}\right] .
$$

It has been shown ${ }^{20}$ that for $n=2^{k}-1$, the TT-DPI method admits the following implementation

$$
\begin{aligned}
\frac{\rho_{n}^{\mathrm{TT}}\left(x, x^{\prime} ; \beta\right)}{\rho_{f p}\left(x, x^{\prime} ; \beta\right)}= & \int_{\mathbb{R}} d a_{1,1} \ldots \int_{\mathbb{R}} d a_{k, 2^{k-1}}(2 \pi)^{-n / 2} \\
& \times \exp \left(-\frac{1}{2} \sum_{l=1}^{k} \sum_{i=1}^{2^{l-1}} a_{l, i}^{2}\right) \\
& \times \exp \left\{-\beta \sum_{i=0}^{2^{k}} \omega_{i} V\left[x_{r}\left(u_{i}\right)\right.\right. \\
& \left.\left.+\sigma \sum_{l=1}^{k} F_{l,\left[2^{l-1} u_{i}\right]+1}\left(u_{i}\right) a_{l,\left[2^{l-1} u_{i}\right]+1}\right]\right\},
\end{aligned}
$$

where $u_{i}=2^{-k} i$ for $0 \leqslant i \leqslant 2^{k}$ and

$$
\omega_{i}=\left\{\begin{array}{l}
2^{-(k+1)}, \quad \text { if } i \in\left\{0,2^{k}\right\}, \\
2^{-k}, \quad \text { if } 1 \leqslant i \leqslant 2^{k}-1 .
\end{array}\right.
$$


The functions $F_{l, k}(u)$ are the so-called Schauder functions, ${ }^{21}$ the definitions of which are presented in the cited references. We leave it for the reader to use Eq. (21) and show that if $x=x^{\prime}$, then all the points $x+\sigma \widetilde{B}_{u_{i}, n}^{0}(\bar{a})$ have identical marginal distribution given by the formula

$$
\frac{\rho_{n}^{\mathrm{TT}}(x ; \beta) d x}{\int_{\mathrm{R}} \rho_{n}^{\mathrm{TT}}(x ; \beta) d x} .
$$

In this case, the point and the path estimators produce identical results for the ensemble average of a diagonal operator $\hat{O}$

$$
\frac{\int_{\mathrm{R}} \rho_{n}^{\mathrm{TT}}(x ; \beta) O(x) d x}{\int_{\mathbb{R}} \rho_{n}^{\mathrm{TT}}(x ; \beta) d x} .
$$

At least for the ensemble average of the potential energy, one should always use the path estimator, which has smaller variance.

A second method for which there is an estimator giving the same values as the point estimator but having (asymptotically, as $n \rightarrow \infty)$ the variance of the path estimator is the so-called Lévy-Ciesielski reweighted technique (RW-LCPI) defined by the formula ${ }^{4}$

$$
\begin{aligned}
\frac{\rho_{n}^{\mathrm{LC}}\left(x, x^{\prime} ; \beta\right)}{\rho_{f p}\left(x, x^{\prime} ; \beta\right)}= & \int_{\mathbb{R}} d a_{1,1} \ldots \int_{\mathbb{R}} d a_{k+2,2^{k+1}}(2 \pi)^{-(4 n+3) / 2} \exp \left(-\frac{1}{2} \sum_{l=1}^{k+2} \sum_{j=1}^{2^{l-1}} a_{l, j}^{2}\right) \\
& \times \exp \left\{-\beta \int_{0}^{1} V\left[x_{r}(u)+\sigma \sum_{l=1}^{k+2} a_{l,\left[2^{l-1} u\right]+1} \widetilde{F}_{l,\left[2^{l-1} u\right]+1}^{(n)}(u)\right] d u\right\},
\end{aligned}
$$

where $\left[2^{l-1} u\right]$ is the integer part of $2^{l-1} u$. It has been shown that for $n=2^{k}-1$, the RW-LCPI method can be put in the Trotter product form ${ }^{4}$

$$
\begin{aligned}
\rho_{n}^{\mathrm{LC}}\left(x, x^{\prime} ; \beta\right)= & \int_{\mathrm{R}} d x_{1} \ldots \int_{\mathrm{R}} d x_{n} \rho_{0}^{\mathrm{LC}}\left(x, x_{1} ; \frac{\beta}{n+1}\right) \\
& \times \ldots \rho_{0}^{\mathrm{LC}}\left(x_{n}, x^{\prime} ; \frac{\beta}{n+1}\right),
\end{aligned}
$$

where

$$
\begin{aligned}
\frac{\rho_{0}^{\mathrm{LC}}\left(x, x^{\prime} ; \beta\right)}{\rho_{f p}\left(x, x^{\prime} ; \beta\right)}= & \frac{1}{(2 \pi)^{3 / 2}} \int_{\mathbb{R}} \int_{\mathrm{R}} \int_{\mathrm{R}} e^{-\left(a_{1}^{2}+a_{2}^{2}+a_{3}^{2}\right) / 2} \\
& \times \exp \left\{-\beta \int_{0}^{1} V\left[x+\left(x^{\prime}-x\right) u\right.\right. \\
& +a_{1} \sigma C_{0}(u)+a_{2} \sigma L_{0}(u) \\
& \left.\left.+a_{3} \sigma R_{0}(u)\right] d u\right\} d a_{1} d a_{2} d a_{3} .
\end{aligned}
$$

The analytical expressions of the functions $\widetilde{F}_{k, l}^{(n)}(u), L_{0}(u)$, $R_{0}(u)$, and $C_{0}(u)$ can be found in Refs. 4 and 6.

Again, we leave it for the reader to use Eq. (24) and prove that if $x^{\prime}=x$, then all the points

$$
x+\sigma \sum_{l=1}^{k+2} a_{l,\left[2^{l-1} u_{i}\right]+1} \widetilde{F}_{l,\left[2^{l-1} u_{i}\right]+1}^{(n)}\left(u_{i}\right),
$$

with $u_{i}=2^{-k} i$ for $0 \leqslant i \leqslant 2^{k}$ have identical marginal distributions equal to that of $x$. The estimator

$$
2^{-k} \sum_{i=0}^{2^{k}-1} O\left[x+\sigma \sum_{l=1}^{k+2} a_{l,\left[2^{l-1} u_{i}\right]+1} \widetilde{F}_{l,\left[2^{l-1} u_{i}\right]+1}^{(n)}\left(u_{i}\right)\right]
$$

produces the same results as the point estimator, but it has the variance of the path estimator. As far as the evaluation of the average potential energy is concerned, in order to avoid unnecessary calls to the potential routine, it is desirable that the points $\left\{2^{-k} i ; 0 \leqslant i \leqslant 2^{k}\right\}$ be among the quadrature points utilized for the computation of the path averages appearing in Eq. (23). The quadrature technique designed in Ref. 6 shares this property. As opposed to the TT-DPI method, the point and the path estimators for the RW-LCPI method produce different results.

\section{B. Estimators for the total energy}

In this subsection, we discuss the implementation of the thermodynamic $(\mathrm{T})$ and the direct $(\mathrm{H})$ estimators for the total energy. The T-method estimator is defined as the following functional of the diagonal density matrix:

$$
\langle E\rangle_{\beta}^{T}=-\frac{\partial}{\partial \beta} \ln \left[\int_{\mathrm{R}} \rho(x ; \beta) d x\right] .
$$

The above formula can be expressed as the statistical average

$$
\langle E\rangle_{\beta}^{T}=\frac{\int_{\mathbb{R}} d x \int_{\Omega} d P[\bar{a}] X_{\infty}(x, \bar{a} ; \beta) E_{\infty}^{T}(x, \bar{a} ; \beta)}{\int_{\mathbb{R}} d x \int_{\Omega} d P[\bar{a}] X_{\infty}(x, \bar{a} ; \beta)},
$$

where the T-method estimating function $E_{\infty}^{T}(x, \bar{a} ; \beta)$ can be shown to be ${ }^{2}$

$$
\begin{aligned}
E_{\infty}^{T}(x, \bar{a} ; \beta)= & \frac{1}{2 \beta}+\int_{0}^{1} V\left[x+\sigma B_{u}^{0}(\bar{a})\right] d u \\
& +\frac{\sigma}{2} \int_{0}^{1} V^{\prime}\left[x+\sigma B_{u}^{0}(\bar{a})\right] B_{u}^{0}(\bar{a}) d u,
\end{aligned}
$$

provided that $e^{-\beta V(x)}$ has (Sobolev) first order derivatives as a function of $x$. For a $d$-dimensional system, the expression of the T-method estimating function reads 


$$
\begin{aligned}
E_{\infty}^{T}\left(x_{1}, \ldots, x_{d}, \bar{a}_{1}, \ldots, \bar{a}_{d} ; \beta\right) & \\
= & \frac{d}{2 \beta}+\int_{0}^{1} V\left[x_{1}+\sigma_{1} B_{u}^{0,1}\left(\bar{a}_{1}\right), \ldots, x_{d}\right. \\
& \left.+\sigma_{d} B_{u}^{0, d}\left(\bar{a}_{d}\right)\right] d u+\sum_{i=1}^{d} \frac{\sigma_{i}}{2} \int_{0}^{1}\left\{\frac { \partial } { \partial x _ { i } } V \left[x_{1}\right.\right. \\
& \left.\left.+\sigma_{1} B_{u}^{0,1}\left(\bar{a}_{1}\right), \ldots, x_{d}+\sigma_{d} B_{u}^{0, d}\left(\bar{a}_{d}\right)\right]\right\} \\
& \times B_{u}^{0, i}\left(\bar{a}_{i}\right) d u .
\end{aligned}
$$

The ensemble average energy can be obtained as the limit $n \rightarrow \infty$ of the sequence

$$
\langle E\rangle_{\beta, n}^{T}=\frac{\int_{\mathbb{R}} d x \int_{\Omega} d P[\bar{a}] X_{n}(x, \bar{a} ; \beta) E_{n}^{T}(x, \bar{a} ; \beta)}{\int_{\mathbb{R}} d x \int_{\Omega} d P[\bar{a}] X_{n}(x, \bar{a} ; \beta)},
$$

where

$$
\begin{aligned}
E_{n}^{T}(x, \bar{a} ; \beta)= & \frac{1}{2 \beta}+\int_{0}^{1} V\left[x+\sigma \widetilde{B}_{u, n}^{0}(\bar{a})\right] d u \\
& +\frac{\sigma}{2} \int_{0}^{1} V^{\prime}\left[x+\sigma \widetilde{B}_{u, n}^{0}(\bar{a})\right] \widetilde{B}_{u, n}^{0}(\bar{a}) d u .
\end{aligned}
$$

The finite-dimensional integral appearing in Eq. (30) can be evaluated by Monte Carlo integration. In the limit $n \rightarrow \infty$, the variance of the estimator is finite because the square of
$E_{\infty}^{T}(x, \bar{a} ; \beta)$ given by Eq. (21) is a well defined function, the average value of which is finite for smooth enough potentials.

A second energy estimator we employ in the present paper is the H-method estimator. This direct estimator is defined by the equation

$$
\langle E\rangle_{\beta}^{H}=\frac{\left.\int_{\mathrm{R}} \hat{H}_{x^{\prime}} \rho\left(x, x^{\prime} ; \beta\right)\right|_{x^{\prime}=x} d x}{\int_{\mathbb{R}} \rho(x ; \beta) d x},
$$

where the Hamiltonian of the system $\hat{H}_{x^{\prime}}$ is assumed to act on the density matrix through the variable $x^{\prime}$. By explicit computation and some integration by parts, the H-method estimator can be expressed as the statistical average

$$
\langle E\rangle_{\beta}^{H}=\frac{\int_{\mathrm{R}} d x \int_{\Omega} d P[\bar{a}] X_{\infty}(x, \bar{a} ; \beta) E_{\infty}^{H}(x, \bar{a} ; \beta)}{\int_{\mathrm{R}} d x \int_{\Omega} d P[\bar{a}] X_{\infty}(x, \bar{a} ; \beta)},
$$

of the estimating function ${ }^{2}$

$$
\begin{aligned}
E_{\infty}^{H}(x, \bar{a} ; \beta)= & \frac{1}{2 \beta}+V(x)+\frac{\hbar^{2} \beta^{2}}{4 m_{0}} \int_{0}^{1} \int_{0}^{1}(u-\tau)^{2} \\
& \times V^{\prime}\left[x+\sigma B_{u}^{0}(\bar{a})\right] V^{\prime}\left[x+\sigma B_{\tau}^{0}(\bar{a})\right] d u d \tau .
\end{aligned}
$$

The H-estimator is properly defined even for potentials that do not have second-order derivatives. For a $d$-dimensional system, the H-method estimating function reads

$$
\begin{aligned}
E_{\infty}^{H}\left(x_{1}, \ldots, x_{d}, \bar{a}_{1}, \ldots, \bar{a}_{d} ; \beta\right)= & \frac{d}{2 \beta}+V\left(x_{1}, \ldots, x_{d}\right)+\sum_{i=1}^{d} \frac{\hbar^{2} \beta^{2}}{4 m_{0, i}} \int_{0}^{1} \int_{0}^{1}(u-\tau)^{2}\left\{\frac{\partial}{\partial x_{i}} V\left[x_{1}+\sigma_{1} B_{u}^{0,1}\left(\bar{a}_{1}\right), \ldots, x_{d}+\sigma_{d} B_{u}^{0, d}\left(\bar{a}_{d}\right)\right]\right\} \\
& \times\left\{\frac{\partial}{\partial x_{i}} V\left[x_{1}+\sigma_{1} B_{\tau}^{0,1}\left(\bar{a}_{1}\right), \ldots, x_{d}+\sigma_{d} B_{\tau}^{0, d}\left(\bar{a}_{d}\right)\right]\right\} d u d \tau .
\end{aligned}
$$

The reader should notice that the double integral appearing in Eq. (34) is really a sum of products of one dimensional integrals. Indeed, one easily computes

$$
\begin{aligned}
E_{\infty}^{H}(x, \bar{a} ; \beta)= & \frac{1}{2 \beta}+V(x)+\frac{\hbar^{2} \beta^{2}}{2 m_{0}} \\
& \times\left\{\int_{0}^{1} u^{2} V^{\prime}\left[x+\sigma B_{u}^{0}(\bar{a})\right] d u\right\} \\
& \times\left\{\int_{0}^{1} V^{\prime}\left[x+\sigma B_{u}^{0}(\bar{a})\right] d u\right\} \\
& -\frac{\hbar^{2} \beta^{2}}{2 m_{0}}\left\{\int_{0}^{1} u V^{\prime}\left[x+\sigma B_{u}^{0}(\bar{a})\right] d u\right\}^{2} .
\end{aligned}
$$

The H-method estimator is the sum of the "classical" energy and a "quantum" correction term. Equation (33) shows that the total energy can also be recovered as the limit $n \rightarrow \infty$ from the sequence

$$
\langle E\rangle_{\beta, n}^{H}=\frac{\int_{\mathrm{R}} d x \int_{\Omega} d P[\bar{a}] X_{n}(x, \bar{a} ; \beta) E_{n}^{H}(x, \bar{a} ; \beta)}{\int_{\mathrm{R}} d x \int_{\Omega} d P[\bar{a}] X_{n}(x, \bar{a} ; \beta)},
$$

where

$$
\begin{aligned}
E_{n}^{H}(x, \bar{a} ; \beta)= & \frac{1}{2 \beta}+V(x)+\frac{\hbar^{2} \beta^{2}}{4 m_{0}} \int_{0}^{1} \int_{0}^{1}(u-\tau)^{2} \\
& \times V^{\prime}\left[x+\sigma \widetilde{B}_{u, n}^{0}(\bar{a})\right] \\
& \times V^{\prime}\left[x+\sigma \widetilde{B}_{\tau, n}^{0}(\bar{a})\right] d u d \tau .
\end{aligned}
$$

The forms of the $\mathrm{T}$ - and the $\mathrm{H}$-method estimators derived here with the reweighted techniques in mind extend naturally to the TT-DPI method by means of Eq. (22). One just replaces the one dimensional integrals appearing in Eqs. (31) and (38) by appropriate trapezoidal quadrature sums.

For the reweighted techniques, we anticipate that the kinetic energy estimator entering the $\mathrm{H}$-method estimator provides more accurate results than the kinetic energy estimator entering the T-method estimator. As for the point and the path estimators of diagonal operators, the derivatives of the 
density matrix against the spatial coordinates, which measure fluctuations around the preferential points $x$ and $x^{\prime}$ for which the reweighted density matrices are optimized, are expected to be reproduced in a better way than the temperature derivatives, which involve unoptimized path-averaged fluctuations. However, for sufficiently low temperatures, the variance of the H-method kinetic energy estimator is expected to be larger than the variance of its thermodynamic counterpart. This larger variance is due to the factor $\beta^{2}$ appearing in Eqs. (34) and (38).

There is one special property of the $\mathrm{T}$ - and H-method estimators that proves to be important in simulations. Let us notice that by virtue of the Bloch equation

$$
\hat{H}_{x^{\prime}} \rho\left(x, x^{\prime} ; \beta\right)=-\frac{\partial}{\partial \beta} \rho\left(x, x^{\prime} ; \beta\right),
$$

we have the equality

$$
\langle E\rangle_{\beta}:=\langle E\rangle_{\beta}^{H}=\langle E\rangle_{\beta}^{T}
$$

Here, the symbol := signifies that the average energy $\langle E\rangle_{\beta}$ is defined to be the common value of the T-method and the $\mathrm{H}$-method energy estimators, provided that these are equal. However, since $\rho_{n}^{\mathrm{RW}}\left(x, x^{\prime} ; \beta\right)$ does not satisfy the Bloch equation (except for the free particle), in general

$$
\begin{aligned}
\langle E\rangle_{\beta, n}^{H} & =\frac{\left.\int_{\mathbb{R}} \hat{H}_{x^{\prime}} \rho_{n}^{\mathrm{RW}}\left(x, x^{\prime} ; \beta\right)\right|_{x^{\prime}=x} d x}{\int_{\mathbb{R}} \rho_{n}^{\mathrm{RW}}(x ; \beta) d x} \\
& \neq\langle E\rangle_{\beta, n}^{T}=-\frac{\partial}{\partial \beta} \ln \left[\int_{\mathbb{R}} \rho_{n}^{\mathrm{RW}}(x ; \beta) d x\right]
\end{aligned}
$$

and the $\mathrm{T}$ - and $\mathrm{H}$-method estimators produce the same result only in the limit $n \rightarrow \infty$. Given that the two energy estimators discussed in the present section can be computed simultaneously without incurring any computational penalty, we recommend that the agreement between the $\mathrm{T}$ - and the $\mathrm{H}$-method estimators be used as a verification tool in actual simulations in order to check the convergence of various path integral methods. However, we emphasize that the agreement between the $\mathrm{T}$ - and the $\mathrm{H}$-method estimators is not a sufficient convergence criterion and in practice, the convergence of different ensemble averages with the number of path variables should also be monitored.

As Eqs. (31) and (38) show, the path and the point estimating functions for the potential energy enter naturally the expressions of the $\mathrm{T}$ - and $\mathrm{H}$-method estimating functions, respectively. For the purpose of using the agreement between the two energy estimators as a verification tool for convergence, one should not replace the path estimating function for the potential energy in the expression of the T-method estimator with the point estimating function, even if this may improve the estimated energy. For special cases, as for instance the TT-DPI and RW-LCPI methods discussed in the previous subsection, one may replace the point estimating function for the potential energy appearing in the expression of the H-method estimator with other estimating functions that produce the same value but have smaller variance. In this paper, we replace the point estimating function with the path estimating function for the TT-DPI method and with the estimating function given by Eq. (25) for the RW-LCPI method, respectively.

\section{A NUMERICAL EXAMPLE}

We have tested the relative merits of the $\mathrm{T}$ - and H-method energy estimators on a cluster of 22 hydrogen molecules at a temperature of $6 \mathrm{~K}$, using three different path integral methods. Two of these methods, the trapezoidal Trotter discrete path integral method and a Lévy-Ciesielski reweighted technique, have been already presented in the preceding section. The third method is a Wiener-Fourier reweighted (RW-WFPI) technique introduced in Ref. 4. The numerical implementation of the methods has been extensively discussed in Ref. 6 by some of us and are not reviewed here.

The physical system we study has been recently examined by Chakravarty, Gordillo, and Ceperley ${ }^{15}$ as well as by Doll and Freeman ${ }^{16}$ in their comparison of Fourier and discrete path integral Monte Carlo methods. The total potential energy of the $\left(\mathrm{H}_{2}\right)_{22}$ cluster is given by

$$
V_{\mathrm{tot}}=\sum_{i<j}^{N} V_{\mathrm{LJ}}\left(r_{i j}\right)+\sum_{i=1}^{N} V_{c}\left(\mathbf{r}_{\mathbf{i}}\right),
$$

where $V_{\mathrm{LJ}}\left(r_{i j}\right)$ is the pair interaction of Lennard-Jones (LJ) potential

$$
V_{\mathrm{LJ}}\left(r_{i j}\right)=4 \epsilon_{\mathrm{LJ}}\left[\left(\frac{\sigma_{\mathrm{LJ}}}{r_{i j}}\right)^{12}-\left(\frac{\sigma_{\mathrm{LJ}}}{r_{i j}}\right)^{6}\right],
$$

and $V_{c}\left(\mathbf{r}_{\mathbf{i}}\right)$ is the constraining potential

$$
V_{c}\left(\mathbf{r}_{\mathbf{i}}\right)=\epsilon_{\mathrm{LJ}}\left(\frac{\left|\mathbf{r}_{\mathbf{i}}-\mathbf{R}_{\mathbf{c m}}\right|}{R_{c}}\right)^{20} .
$$

The values of the Lennard-Jones parameters $\sigma_{\mathrm{LJ}}$ and $\epsilon_{\mathrm{LJ}}$ used are $2.96 \AA$ and $34.2 \mathrm{~K}$, respectively. ${ }^{15} \mathbf{R}_{\mathbf{c m}}$ is the coordinate of the center of mass of the cluster and is given by

$$
\mathbf{R}_{\mathbf{c m}}=\frac{1}{N} \sum_{i=1}^{N} \mathbf{r}_{\mathbf{i}}
$$

Finally, $R_{c}=4 \sigma_{\mathrm{LJ}}$ is the constraining radius. The role of the constraining potential $V_{c}\left(\mathbf{r}_{\mathbf{i}}\right)$ is to prevent molecules from permanently leaving the cluster since the cluster in vacuum at any finite temperature is metastable with respect to evaporation.

At the temperature of $6 \mathrm{~K}$ and at the small densities employed in our computation, the molecules of hydrogen can be described by spherical rotational wave functions, because the majority of the molecules are in the $J=0$ state. To a good approximation, the molecules can be regarded as spherical bosons interacting through isotropic pair potentials. However, a thorough study of parahydrogen clusters has showed that quantum exchange of molecules is small at temperatures greater than $2 \mathrm{~K}$ and that the hydrogen molecules can be safely treated as distinguishable particles. ${ }^{22}$

The optimal choice of the parameter $R_{c}$ for the constraining potential has been discussed in recent work. ${ }^{23}$ If $R_{c}$ is taken to be too small, the properties of the system become 
sensitive to its choice, whereas large values of $R_{c}$ can result in problems attaining an ergodic simulation. To facilitate comparisons, in the current work, $R_{c}$ has been chosen to be identical to that used in Ref. 15. While this choice of constraining potential can induce ergodicity problems in calculations of fluctuation quantities like the heat capacity, we provide evidence below that the simulations in the current work are ergodic.

The three path integral methods we have employed utilize different numbers of path variables for a given index $n$. For instance, the TT-DPI $n$th order approximation to the density matrix $\rho_{n}^{\mathrm{TT}}\left(x, x^{\prime} ; \beta\right)$ utilizes $n$ path variables for each physical dimension, whereas $\rho_{n}^{\mathrm{LC}}\left(x, x^{\prime} ; \beta\right)$ and $\rho_{n}^{\mathrm{WF}}\left(x, x^{\prime} ; \beta\right)$ utilize $4 n+3$ and $4 n$ path variables, respectively. To ensure fair comparison with respect to the number of path variables employed, we have tabled the total number of variables $n_{v}$ used for each physical dimension and not the index $n$.

\section{A. Sampling strategy}

We have discussed in Sec. II that the evaluation of the ensemble average of any observable eventually reduces to the evaluation of the average of a certain estimating function against the probability distribution

$$
\frac{X_{n}(x, \bar{a} ; \beta) d x d P[\bar{a}]}{\int_{\mathbb{R}} d x \int_{\Omega} d P[\bar{a}] X_{n}(x, \bar{a} ; \beta)}
$$

or its multidimensional counterpart. This probability distribution can be sampled with the help of the Metropolis algorithm, which comprises the following steps. ${ }^{24,25}$ One initializes the imaginary-time paths in some fashion. Then, one attempts a trial move of the paths, which may involve changing several coordinates at a time. The displacement of the new paths is usually chosen to be relative to the old paths. To ensure ergodicity, one makes sure that all variables of the system are eventually moved in a cyclic or a random fashion. The proposed path is then accepted or rejected with a certain probability. The average value of the quantity of interest is computed by averaging the values of the corresponding estimating function evaluated at the current paths.

To establish some notation necessary for our discussion, for each vector $\mathbf{r}_{i}=\left(x_{i}, y_{i}, z_{i}\right)$ denoting the physical coordinates of the particle $i$, we let $\overline{\mathbf{a}}_{i}=\left\{\mathbf{a}_{i, 1}, \ldots, \mathbf{a}_{i, n_{v}}\right\}$ be the collection of path variables associated with the respective particle. Each

$$
\mathbf{a}_{i, k}=\left(a_{i, k}^{x}, a_{i, k}^{y}, a_{i, k}^{z}\right)
$$

is itself a three-dimensional vector whose components denote the $k$ th parameter of particle $i$ for the $x, y$, and $z$ coordinates, respectively. Going back to the description of the Metropolis algorithm, the full imaginary-time path has been initialized by choosing the physical coordinates $\mathbf{r}_{\mathbf{i}}$ randomly in a sphere of radius $R_{c}$ centered about origin. The path variables $\overline{\mathbf{a}}_{\mathbf{i}}$ have been initialized with zero.

Except for the Wiener-Fourier method with $n_{v}=512$ $(n=128)$, we update the individual particles one at a time in a cyclic fashion. Each update of a particle consists of an attempt to move the physical coordinate $\mathbf{r}_{i}$ together with the first one quarter of the path variables $\overline{\mathbf{a}}_{i}$ (that is, together with the variables $\left.\left\{\mathbf{a}_{i, k} ; 1 \leqslant k \leqslant\left[n_{v} / 4\right]\right\}\right)$ followed by a separate attempt to move the rest of the path variables associated with the particle $i$. Both the physical coordinates and the path variables are moved in a cube centered about the old coordinates:

$$
\mathbf{r}_{i}^{\prime}=\mathbf{r}_{i}+\Delta_{r}(2 \mathbf{u}-1)
$$

and

$$
\mathbf{a}_{i, k}^{\prime}=\mathbf{a}_{i, k}+\Delta_{a}(2 \mathbf{u}-1),
$$

where the three components of $\mathbf{u}$ are independent uniformly distributed random numbers on the interval $[0,1]$. Throughout our simulations, we have used the following maximum displacement values: $\Delta_{r}=0.26 \AA$ and $\Delta_{a}=0.15$. The sampling technique employed guarantees an acceptance ratio between $30 \%$ and $70 \%$ for all methods studied and for $n_{v}$ $\leqslant 256$.

Because the acceptance ratio drops below $20 \%$ for the Wiener-Fourier reweighted technique with $n_{v}=512$, each most basic step of the previously described algorithm has been decomposed into two successive steps. The first step is decomposed into an attempt to move the physical coordinate $\mathbf{r}_{i}$ together with the first $1 / 8$ of the path variables $\overline{\mathbf{a}}_{i}$, followed by an attempt to move the physical coordinate $\mathbf{r}_{i}$ together with the next $1 / 8$ path variables $\overline{\mathbf{a}}_{i}$. The second step is decomposed in a similar fashion; half of the remaining variables have been moved in a first attempt and then the other half in a second attempt. This restores the overall acceptance ratio to about $33 \%$. In fact, we have monitored separately the acceptance ratio for the four different steps necessary to update all the coordinates associated with a given particle and have made sure that the sampling is well balanced in the sense that the acceptance for each individual step is about $30 \%$ or larger.

As a counting device, we define a pass as the minimal set of Monte Carlo attempts over all variables in the system. A pass consists of $2 \cdot 22=44$ basic steps for all simulations with $n_{v} \leqslant 256$. For the Wiener-Fourier reweighted technique with $n_{v}=512$, a pass consists of $4 \cdot 22=88$ basic attempted moves. One also defines a block as a computational unit made up of ten thousand passes.

\section{B. Embarrassingly parallel computation}

In order to achieve a statistical error of about 0.1 $\mathrm{K} /$ molecule for all computed energies, we have employed a large number of Monte Carlo passes (10.4 million) and we have divided the computation in 16 independent tasks to be run in parallel. For the Wiener-Fourier reweighted method with $n_{v}=512$, we have utilized a number of 40 million passes divided in 80 independent tasks. The Monte Carlo simulations are embarrassingly parallel provided that one can generate independent streams of uniformly distributed random numbers. In this situation, there is no need for communication among the different code replica running on different nodes, and the program is an ideal candidate for use on a distributed computing cluster. However, to be mathematically rigorous, it is necessary to ensure that all the communication needed is already buried in the independence of the streams of random numbers. This underlies the need for "good" parallel random number generators. 
The Mersenne twister (MT) is a fast serial pseudorandom number generating algorithm with a long period and good $k$-distribution properties. ${ }^{26}$ Quite interestingly, the algorithm allows for the development of random number generators meeting certain user specifications. For instance, one may specify the period (which must be a Mersenne prime number, i.e., a prime number of the form $2^{p}-1$ ), the word size, or the memory size. Given a specified period, one may still produce various algorithms which differ by their characteristic polynomials. The dynamic creation of distributed random number generators is based on the hypothesis that MT random number generators having relatively prime characteristic polynomials produce highly independent streams of random numbers. ${ }^{27}$ Because the laws by which the numbers are generated are significantly different, it is very probable that the streams produced by the different generators are highly uncorrelated. In this paper, we have used the Dynamic Creator C-language library ${ }^{28}$ with the Mersenne number $2^{3217}$ -1 . The library outputs streams of 32-bit integers, which are easy to convert into real numbers on the interval $[0,1]$. Different streams are identified by different identification numbers. The streams have been initialized once at the beginning of the simulation with different seeds.

Given the 16 streams of independent random numbers, the Monte Carlo simulation proceeds as follows. For each stream, one performs an independent simulation consisting of 65 blocks. These blocks are preceded by 13 equilibration blocks, which are needed to bring the system into probable configurations but do not contribute to the averages of the estimating functions. For the Wiener-Fourier reweighted method with $n_{v}=512$, we use 80 independent streams of 50 blocks each, for a total of 40 million passes. The equilibration phase consists of 10 blocks for each stream. Ideally, the length of the individual streams should be chosen to be sufficiently large, that the averages of the computed property for different streams are independent and normally distributed, as dictated by the central limit theorem. This requirement is satisfied by all simulations we have performed.

We have collected individual averages for all blocks and streams and performed several statistical tests verifying the applicability of the central limit theorem as well as the independence between the block averages of same or different streams. Let $\left\{Z_{i, j}: 1 \leqslant i \leqslant 16 ; 1 \leqslant j \leqslant 65\right\}$ denote the blockaverages of the property $Z$ for stream $i$ and block $j$ (the RW-WFPI simulation for $n_{v}=512$ has been analyzed in a similar fashion). Under the assumption that the size of the blocks is large enough so that the correlation between different block-averages is negligible and under the assumption that the block-averages for different streams are highly uncorrelated, the values $Z_{i, j}$ should have a Gaussian distribution centered around the average value

$$
\bar{Z}=\frac{1}{16 \cdot 65} \sum_{i=1}^{16} \sum_{j=1}^{65} Z_{i, j},
$$

with variance

$$
\sigma^{2}(Z)=\frac{1}{16 \cdot 65}\left(\sum_{i=1}^{16} \sum_{j=1}^{65} Z_{i, j}^{2}\right)-\bar{Z}^{2} .
$$

The validity of this assumption can be verified with the help of the Shapiro-Wilks normality test. ${ }^{29}$ If the collection of samples $Z_{i, j}$ does not pass the test, it does not necessarily follow that the samples $Z_{i, j}$ are not independent, as their distribution is normal only if the size of the blocks is sufficiently large. At a significance level of 5\%, we do not reject the Gaussian distribution hypothesis for all computed average properties. To within the statistical significance of our calculations, the samples $Z_{i, j}$ can be assumed to be independent and have a Gaussian distribution.

A second set of tests consists in verifying that the row and column averages of $Z_{i, j}$ have Gaussian distributions centered around $\bar{Z}$ with variances $\sigma^{2}(Z) / 65$ and $\sigma^{2}(Z) / 16$, respectively. The validity of this distribution follows from the central limit theorem and the assumption that the samples $Z_{i, j}$ are independent and have a Gaussian distribution characterized by the average $\bar{Z}$ and the variance $\sigma^{2}(Z)$. It is important to emphasize that the row averages must pass this test. As previously discussed, the number of blocks in a stream should be sufficiently large so that the row averages have the required distribution even if the independent samples $Z_{i, j}$ do not have a Gaussian distribution. Again, under the assumption of independence only, the row averages should have a Gaussian distribution centered around $\bar{Z}$ and have variance $\sigma^{2}(Z) / N_{\text {blocks }}$ for a sufficiently large number of blocks $N_{\text {blocks }}$. We have employed the KolmogorovSmirnov test ${ }^{30}$ to compare the distributions of the row and column averages with the theoretical Gaussian distributions. For all computed average properties, we find that the respective distributions are identical at a statistical significance level of 5\%. The agreement for the distribution of the row averages is evidence that the streams generated by the Dynamic Creator package are sufficiently independent, whereas the agreement for the distribution of the column averages is evidence that the block averages of the same streams are independent.

For the third set of tests, we have considered two timeseries $\left\{Z_{i}^{\prime}, 1 \leqslant i \leqslant 16 \cdot 65\right\}$ and $\left\{Z_{i}^{\prime \prime}, 1 \leqslant i \leqslant 16 \cdot 65\right\}$ obtained by concatenating the rows of the matrix $Z_{i, j}$ and the columns, respectively. We then have studied the autocorrelation of the two time series for a maximum lag of 32 . The correlation coefficients for a lag $k \leqslant 32$ are computed with the formula

$$
r_{k}^{\prime}=\frac{1}{\sigma^{2}(Z)} \frac{1}{16 \cdot 65} \sum_{i=1}^{16 \cdot 65}\left(Z_{i}^{\prime}-\bar{Z}\right)\left(Z_{i+k}^{\prime}-\bar{Z}\right),
$$

where $Z_{i+k}^{\prime}=Z_{i+k-16.65}^{\prime}$ if $i+k>16 \cdot 65$. Under the independence hypothesis of the samples $Z_{i}^{\prime}$, the statistics of the correlation coefficients for $1 \leqslant k \leqslant 32$ is normal with average zero and standard deviation $\sigma^{\prime}=1 / \sqrt{16 \cdot 65}$. Moreover, the correlation coefficients can be regarded as independent samples of this normal distribution. By the binomial distribution, the probability that at most $m$ correlation coefficients lie outside the interval $\left[-2 \sigma^{\prime}, 2 \sigma^{\prime}\right]$ is given by the formula

$$
P(m)=\sum_{k=0}^{m} \frac{32 !}{k !(32-k) !} q^{k}(1-q)^{32-k},
$$

where $q \approx 0.046$ is the probability that a normal distributed variable of mean zero and standard deviation $\sigma^{\prime}$ lies outside 

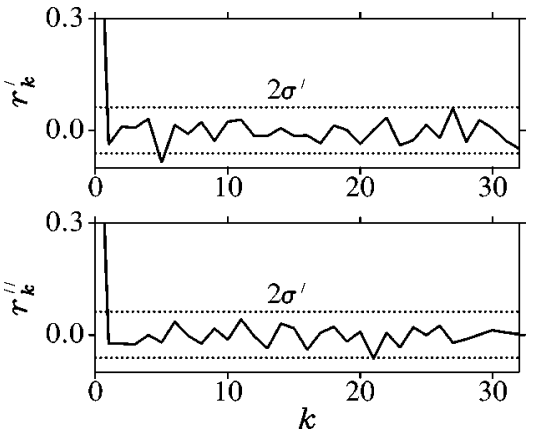

FIG. 1. Correlograms for the time-series $Z_{i}^{\prime}$ and $Z_{i}^{\prime \prime}$. The property $Z$ is the average ensemble energy computed by means of the H-method estimator using the RW-WFPI method with $n_{v}=32$. One notices that both the correlation between the block averages $\left(r_{k}^{\prime}\right)$ and the correlation between the streams $\left(r_{k}^{\prime \prime}\right)$ are negligible.

the interval $\left[-2 \sigma^{\prime}, 2 \sigma^{\prime}\right]$. One computes $P(3)=0.942$ and $P(4)=0.985$ so at a level of significance of $5 \%$, the hypothesis that the $r_{k}^{\prime}$ are independent samples of a normally distributed variable of mean zero and standard deviation $\sigma^{\prime}$ $=1 / \sqrt{16 \cdot 65}$ should be rejected if 4 or more correlation coefficients lying outside the interval $\left[-2 \sigma^{\prime}, 2 \sigma^{\prime}\right]$ are observed.

The autocorrelation of the series $Z_{i}^{\prime}$ is representative of the correlation between the block averages of same streams, whereas the autocorrelation of the time series $Z_{i}^{\prime \prime}$ is representative of the correlation between the blocks of similar rank corresponding to different streams. Figure 1 shows the correlograms of the two series for a RW-WFPI Monte Carlo simulation with $n_{v}=32$. The computed property is the $\mathrm{H}$-method energy estimator. Both series $Z_{i}^{\prime}$ and $Z_{i}^{\prime \prime}$ have only one point lying outside the interval $\left[-2 \sigma^{\prime}, 2 \sigma^{\prime}\right]$. These points are $r_{5}^{\prime}$ and $r_{21}^{\prime \prime}$, respectively (of course, the points $r_{0}^{\prime}$ $=r_{0}^{\prime \prime}=1$ are not counted). Consequently, the simulation passes our third statistical test. In fact, all the simulations performed have passed this statistical test for all computed properties. We conclude that the correlation between the block averages of same or different streams is negligible. By the central limit theorem, the statistical error in the determination of the average of the property $Z$ is

$$
\pm 2 \sigma(Z) / \sqrt{16 \cdot 65}
$$

where $\sigma^{2}(Z)$ is defined by Eq. (45). (For the statistical error, we employ the $2 \sigma$ value, corresponding to an interval of $95 \%$ confidence. The $5 \%$ probability that the results lie outside the confidence interval is chosen to agree with the level of significance of the statistical tests).

The analysis performed in the present subsection demonstrates that the streams generated by the Dynamic Creator algorithm have negligible correlation at least for our purposes.

A separate advantage in the use of independent streams is to overcome the phenomenon of quasiergodicity, ${ }^{31}$ which might appear in Monte Carlo simulations whenever the distribution that is sampled has several well defined minima that are separated by walls of high energy. In this case, the random walker may be trapped in one of the wells and never sample the others, or sample them with the wrong frequency. The Monte Carlo simulation may pass all the aforementioned statistical tests but still produce the wrong results. For our system, the probability that such a situation may occur is quite low because the system is highly quantum mechanical with strong barrier tunneling. Moreover, the 16 independent streams have been initialized randomly in configuration space. This makes it unlikely that all the streams are trapped precisely into the same local minimum or group of local minima. Evidence for quasiergodicity may be captured in the form of a few outlying averages among the stream averages. Such outlying averages have not been observed.

\section{Summary and discussion of the computed averages}

The computed averages for all methods and estimators utilized are presented in Tables I-III. For a given number of path variables $n_{v}$, the RW-WFPI, RW-LCPI, and TT-DPI methods utilize $2 n_{v}, 2.25 n_{v}$, and $n_{v}$ quadrature points, respectively. (For a discussion of the minimal number of quadrature points and of the nature of the quadrature schemes that must be employed for the first two methods, the reader should consult Ref. 6. For the RW-WFPI method, we have utilized $2 n_{v}$ Gauss-Legendre quadrature points, though a number of $1.75 n_{v}$ points would have sufficed.) The observed overall computational time for the three methods have followed the ratios $2: 2.25: 1$, even though the time necessary to compute the paths is proportional to $n_{v}^{2}$ for the first method and to $n_{v} \log _{2}\left(n_{v}\right)$ for the other methods. The computation of the paths takes full advantage of the vector floating point units of the modern processors and is dominated by the calls to the potential, except for very large $n_{v}$.

TABLE I. Listed are the results obtained by the Wiener-Fourier reweighted path integral method. Average potential $\langle V\rangle_{\beta}$, kinetic $\langle K\rangle_{\beta}$, and total energies $\langle E\rangle_{\beta}$ are calculated with the help of the T- and H-estimators as functions of the number of path variables $n_{v}$. The error bars are two standard deviation values. All energies are given in $\mathrm{K} /$ molecule.

\begin{tabular}{ccccccc}
\hline \hline$n_{v}$ & $\langle E\rangle_{\beta}^{T}$ & $\langle E\rangle_{\beta}^{H}$ & $\langle V\rangle_{\beta}^{T}$ & $\langle V\rangle_{\beta}^{H}$ & $\langle K\rangle_{\beta}^{T}$ & $\langle K\rangle_{\beta}^{H}$ \\
\hline 4 & $-57.66 \pm 0.05$ & $-16.63 \pm 0.18$ & $-82.14 \pm 0.07$ & $-61.72 \pm 0.12$ & $24.48 \pm 0.02$ & $45.09 \pm 0.15$ \\
8 & $-37.61 \pm 0.05$ & $-17.77 \pm 0.16$ & $-64.74 \pm 0.06$ & $-53.07 \pm 0.11$ & $27.13 \pm 0.02$ & $35.29 \pm 0.13$ \\
16 & $-25.68 \pm 0.04$ & $-18.28 \pm 0.13$ & $-54.27 \pm 0.06$ & $-49.33 \pm 0.10$ & $28.60 \pm 0.03$ & $31.06 \pm 0.11$ \\
32 & $-20.23 \pm 0.04$ & $-18.00 \pm 0.12$ & $-49.66 \pm 0.06$ & $-48.05 \pm 0.10$ & $29.42 \pm 0.03$ & $30.05 \pm 0.11$ \\
64 & $-18.29 \pm 0.04$ & $-17.85 \pm 0.11$ & $-48.19 \pm 0.06$ & $-47.86 \pm 0.09$ & $29.90 \pm 0.03$ & $30.01 \pm 0.11$ \\
128 & $-17.75 \pm 0.04$ & $-17.64 \pm 0.12$ & $-47.83 \pm 0.06$ & $-47.81 \pm 0.09$ & $30.08 \pm 0.03$ & $30.17 \pm 0.11$ \\
256 & $-17.71 \pm 0.04$ & $-17.70 \pm 0.12$ & $-47.85 \pm 0.07$ & $-47.87 \pm 0.10$ & $30.14 \pm 0.03$ & $30.17 \pm 0.12$ \\
\hline \hline
\end{tabular}


TABLE II. Listed are the results obtained by the Lévy-Ciesielski reweighted path integral method. The format is that of Table I.

\begin{tabular}{ccccccc}
\hline \hline$n_{v}$ & $\langle E\rangle_{\beta}^{T}$ & $\langle E\rangle_{\beta}^{H}$ & $\langle V\rangle_{\beta}^{T}$ & $\langle V\rangle_{\beta}^{H}$ & $\langle K\rangle_{\beta}^{T}$ & $\langle K\rangle_{\beta}^{H}$ \\
\hline 3 & $-70.46 \pm 0.06$ & $18.24 \pm 0.20$ & $-93.47 \pm 0.07$ & $-69.03 \pm 0.09$ & $23.01 \pm 0.02$ & $87.27 \pm 0.19$ \\
7 & $-44.08 \pm 0.05$ & $-10.81 \pm 0.15$ & $-71.03 \pm 0.06$ & $-55.08 \pm 0.08$ & $26.94 \pm 0.02$ & $44.28 \pm 0.14$ \\
15 & $-29.84 \pm 0.04$ & $-15.84 \pm 0.12$ & $-58.33 \pm 0.06$ & $-49.10 \pm 0.07$ & $28.50 \pm 0.02$ & $33.26 \pm 0.12$ \\
31 & $-22.76 \pm 0.04$ & $-17.40 \pm 0.10$ & $-51.95 \pm 0.06$ & $-47.83 \pm 0.06$ & $29.19 \pm 0.03$ & $30.43 \pm 0.11$ \\
63 & $-19.50 \pm 0.04$ & $-17.68 \pm 0.10$ & $-49.15 \pm 0.06$ & $-47.69 \pm 0.06$ & $29.65 \pm 0.03$ & $30.01 \pm 0.11$ \\
127 & $-18.25 \pm 0.04$ & $-17.68 \pm 0.10$ & $-48.20 \pm 0.06$ & $-47.80 \pm 0.06$ & $29.95 \pm 0.03$ & $30.11 \pm 0.11$ \\
255 & $-17.84 \pm 0.04$ & $-17.65 \pm 0.11$ & $-47.93 \pm 0.07$ & $-47.85 \pm 0.07$ & $30.09 \pm 0.03$ & $30.20 \pm 0.12$ \\
\hline \hline
\end{tabular}

As discussed in Ref. 6, the asymptotic convergence for the reweighted techniques is expected to be cubic, even for the Lennard-Jones potential that is not included in the class of potentials for which cubic convergence has been demonstrated formally. We find that the asymptotic convergence is attained only for very large $n_{v}$, as one may see by comparing for example the total, potential, and kinetic energies computed with the help of the T-method estimator for the RW-LCPI and the TT-DPI methods. Even if the latter method has only $1 / n_{v}^{2}$ asymptotic convergence, the two methods produce almost equal results. In fact, a numerical analysis of the relationship

$$
\langle E\rangle_{\beta, n_{v}}^{T} \approx\langle E\rangle_{\beta}+\frac{\text { const }}{\left(n_{v}\right)^{\alpha}},
$$

in which the left-hand side quantity is plotted against $1 /\left(n_{v}\right)^{\alpha}$ for different values of $\alpha$, suggests that, while the methods have converged within the statistical error, none of the three methods includes sufficiently large values of $n_{v}$ to attain the ultimate asymptotic rate of convergence.

When comparing the values of the H-method energy estimator and of the related potential and kinetic estimators for the three path integral techniques, one notices that the RWLCPI technique provides better values than the TT-DPI method. The H-method estimator has a better behavior if used together with a reweighted technique. This behavior is consistent with the analysis we have performed in Sec. II on the values of the potential point-estimators and the excellent values found with the RW-WFPI method. For the reweighted techniques, the $\mathrm{H}$-method estimator provides better energy values than the T-method estimator. This is also true of the potential and kinetic parts of the estimators. However, the variance of the H-method estimator is significantly larger than the variance of the T-method estimator and the difference is even more pronounced if one compares the corresponding kinetic estimators.

As discussed in Sec. II A, the path estimator for the potential energy has a smaller variance than the point estimator. Indeed, the results from Table I show that the variance of the path estimator is approximately $(0.9 / 0.6)^{2}=2.25$ times smaller than the variance of the point estimator. In the case of the RW-LCPI and TT-DPI methods, we have employed the estimator given by Eq. (25) and the path estimator, respectively. These were shown to produce values identical to the point estimator but have the variance of the path estimator. For the RW-WFPI and RW-LCPI methods, the point and the path estimators produce different results. Due to the very design of the reweighted techniques, we have argued that the point estimator results should be the more accurate ones. This theoretical prediction is well supported by the values presented in Tables I and II.

While we have argued that the $\mathrm{H}$-method estimator is a better estimator as value (but not necessarily as variance) than the T-method estimator for the reweighted methods, it is apparent from Table III that the same difference persists for the trapezoidal Trotter scheme. As discussed before, for the TT-DPI method, the point and path estimators provide the same value for the average potential. As opposed to the reweigthed techniques, the $\mathrm{H}$-method kinetic estimator is less accurate than the T-method kinetic energy estimator. Quite interestingly, even if individually the potential and the kinetic parts are more accurate for the T-method estimator, it is the H-method energy estimator that provides a more accurate total energy. Clearly, a strong compensation of errors appears in the case of the H-method estimator. Such a compensation of errors is generally characteristic of variational methods. In

TABLE III. Listed are the results obtained by the trapezoidal Trotter discrete path integral method. The format is that of Table I.

\begin{tabular}{ccccccc}
\hline \hline$n_{v}$ & $\langle E\rangle_{\beta}^{T}$ & $\langle E\rangle_{\beta}^{H}$ & $\langle V\rangle_{\beta}^{T}$ & $\langle V\rangle_{\beta}^{H}$ & $\langle K\rangle_{\beta}^{T}$ & $\langle K\rangle_{\beta}^{H}$ \\
\hline 3 & $-68.54 \pm 0.05$ & $78.08 \pm 0.30$ & $-89.88 \pm 0.07$ & $-89.88 \pm 0.07$ & $21.34 \pm 0.02$ & $167.97 \pm 0.32$ \\
7 & $-45.29 \pm 0.05$ & $7.22 \pm 0.19$ & $-70.88 \pm 0.06$ & $-70.88 \pm 0.06$ & $25.58 \pm 0.02$ & $78.10 \pm 0.21$ \\
15 & $-30.61 \pm 0.04$ & $-12.52 \pm 0.13$ & $-58.53 \pm 0.06$ & $-58.53 \pm 0.06$ & $27.92 \pm 0.02$ & $46.01 \pm 0.15$ \\
31 & $-22.95 \pm 0.04$ & $-16.86 \pm 0.11$ & $-51.99 \pm 0.06$ & $-51.99 \pm 0.06$ & $29.04 \pm 0.03$ & $35.14 \pm 0.12$ \\
63 & $-19.55 \pm 0.04$ & $-17.66 \pm 0.10$ & $-49.19 \pm 0.06$ & $-49.19 \pm 0.06$ & $29.65 \pm 0.03$ & $31.53 \pm 0.11$ \\
127 & $-18.29 \pm 0.04$ & $-17.70 \pm 0.10$ & $-48.27 \pm 0.06$ & $-48.27 \pm 0.06$ & $29.97 \pm 0.03$ & $30.57 \pm 0.11$ \\
255 & $-17.86 \pm 0.04$ & $-17.71 \pm 0.11$ & $-47.94 \pm 0.07$ & $-47.94 \pm 0.07$ & $30.07 \pm 0.03$ & $30.23 \pm 0.12$ \\
\hline \hline
\end{tabular}


TABLE IV. Estimated energies in K/molecule for the $\left(\mathrm{H}_{2}\right)_{22}$ cluster computed with the help of the Wiener-Fourier reweighted technique using 512 path variables and 40 million Monte Carlo passes. Listed are the average potential $\langle V\rangle_{\beta}$, kinetic $\langle K\rangle_{\beta}$, and total energies $\langle E\rangle_{\beta}$ calculated with the help of the T-method (left column) and H-method (right column) estimators. The reported errors are two standard deviations.

\begin{tabular}{lrrr}
\hline \hline$\langle E\rangle_{\beta}^{T}$ & $-17.69 \pm 0.02$ & $\langle E\rangle_{\beta}^{H}$ & $-17.71 \pm 0.06$ \\
$\langle V\rangle_{\beta}^{T}$ & $-47.82 \pm 0.03$ & $\langle V\rangle_{\beta}^{H}$ & $-47.81 \pm 0.05$ \\
$\langle K\rangle_{\beta}^{T}$ & $30.13 \pm 0.02$ & $\langle K\rangle_{\beta}^{H}$ & $30.10 \pm 0.06$ \\
\hline \hline
\end{tabular}

this respect, notice that the TT-DPI density matrices are positive definite because they are obtained by Lie-Trotter composing a certain symmetrical short-time approximation. By the Ritz variational principle, the H-method energy estimator cannot have a value smaller than the ground-state energy. Thus, the Ritz variational principle provides some control on the values of the H-method estimator, but not on the individual components, nor on the T-method estimator. The RWLCPI density matrices are also positive definite for $n \geqslant 2$ and indeed, the energy provided by the H-method estimator is still better than what the values of the potential and kinetic parts suggest. While a final resolution awaits further study, it is apparent that this finding is not related to the asymptotic rate of convergence of the path integral technique.

Among the three methods presented, the RW-WFPI has the fastest convergence for all properties studied. Moreover, for $n_{v}=128$ and $n_{v}=256$, there is a good agreement (within statistical noise) between the $\mathrm{T}$ - and the H-method energy estimators, as well as between their potential and kinetic energy components. For $n_{v}=256$, one concludes that the systematic error is smaller than the statistical error for all properties computed. An additional RW-WFPI simulation with $n_{v}=512$ in 40 million Monte Carlo passes has produced results consistent with the findings above. The results are summarized in Table IV and represent the energy values we report.

\section{CONCLUSIONS}

In the present work we have considered a number of issues related to the choice of estimators for random series path integral methods. We have illustrated our results by applying them to the problem of computing various thermodynamic properties of a model of the $\left(\mathrm{H}_{2}\right)_{22}$ cluster using reweighted path integral techniques. The molecular hydrogen cluster is a strongly quantum mechanical system and is representative of the type of problems one is likely to encounter in many applications. Hence, it constitutes a useful benchmark for present and future path integral techniques and for this reason it is important that its physical properties be determined within advertized statistical error bars. Path integral methods capable of dealing with such highly quantummechanical systems in an efficient manner are needed, both for reliable determinations of the physical properties of the respective systems as well as for accurate parameterizations of the intermolecular potentials.

We wish to make a number of points concerning the present results and the methods we have utilized to obtain them. At a more general level, we would like to emphasize that the reweighted path integral methods discussed here provide a broadly applicable, simple, and formally well characterized set of techniques. As demonstrated by the present results, they are capable of producing high-quality numerical results for problems of appreciable physical complexity. Moreover, they do so without the assumption of a particular form for the underlying microscopic forces. Furthermore, the estimators described in the present paper are convenient, accurate, and easily implemented for any random series approach. As discussed in Sec. III, when used together, the T and H-method estimators provide an important consistency check on the quality of the path integral simulations. Such consistency checks are a valuable element in judging the reliability of particular simulations.

As previously mentioned, the cluster application discussed here provides a convenient test bed for the development of numerical methods. For this reason, we have exercised due diligence with respect to the quality of our final results summarized in Table IV. As discussed in Sec. III, we have subjected both the parallel random number generator employed and the numerical results obtained to a series of quality-control tests. Beyond these statistical checks, it is important to note there is an internal consistency check on the quality of the present results. Specifically, as is evident in Tables I-III, the kinetic, potential, and total energies from the three different path integral approaches (trapezoidal Trotter, reweighted Lévy-Ciesielski, and reweighted WienerFourier) all agree. It is also important to note in this context that, while the presently computed total energies agree with those reported by Chakravarty et al., ${ }^{15}$ the individual kinetic and potential energies do not. The kinetic energy reported by Chakravarty et al. ${ }^{15}$ is $\sim 0.8 \mathrm{~K} /$ particle higher than found in the present simulations (with the potential energy being correspondingly lower). The magnitude of this difference is well outside the statistical error bars involved and appears to signal a systematic error. Based on the observed consistency between the results produced by three different path integral methods and on the agreement between the $\mathrm{T}$ and $\mathrm{H}$-method estimators for each of these path integral formulations, we feel confident of the results we have reported in Table IV.

Note: After the present simulations had been completed, we have learned from D. M. Ceperley that the off-diagonal pair density used as the starting point in the simulations reported in Ref. 15 was truncated at first order in the expansion of off-diagonal displacements instead of second order and that the inclusion of this second-order term resolves the kinetic and potential energy difference noted above.

\section{ACKNOWLEDGMENTS}

The authors acknowledge support from the National Science Foundation through award Nos. CHE-0095053 and CHE-0131114. They also wish to thank the Center for Advanced Scientific Computing and Visualization (TCASCV) at Brown University, especially Dr. James O'Dell, for valuable assistance with respect to the numerical simulations described in the present paper. They would also like to thank Mr. Cristian Diaconu for helpful discussions concerning the present work. Finally, the authors would like to express a special thanks to Professor David Ceperley for continuing 
discussions concerning the present simulations and for his efforts in tracking down the origin of the pair density issues noted in Sec. IV.

\section{APPENDIX: EQUIVALENCE OF PATH AND POINT ESTIMATORS}

The main purpose of this section is to give a compact form for the integral

$$
\int_{\Omega} d P[\bar{a}] X_{\infty}\left(x, x^{\prime}, \bar{a} ; \beta\right) O\left[x_{r}(\theta)+\sigma B_{\theta}^{0}(\bar{a})\right],
$$

where $\theta$ is an arbitrary point in the interval $[0,1]$. In terms of a standard Brownian motion [see Eq. (1)], the above integral can be put into the form

$$
\begin{aligned}
& P\left[\sigma B_{1}=x^{\prime} \mid \sigma B_{0}=x\right] \mathbb{E}\left[e^{-\beta \int_{0}^{1} V\left(\sigma B_{u}\right) d u} O\left(\sigma B_{\theta}\right) \mid \sigma B_{1}=x^{\prime},\right. \\
& \left.\sigma B_{0}=x\right] \\
& =\int_{\mathbb{R}} O(y) P\left[\sigma B_{1}=x^{\prime}, \sigma B_{\theta}=y \mid \sigma B_{0}=x\right] \\
& \quad \times \mathbb{E}\left[e^{-\beta \int_{0}^{1} V\left(\sigma B_{u}\right) d u} \mid \sigma B_{1}=x^{\prime}, \sigma B_{\theta}=y, \sigma B_{0}=x\right] d y .
\end{aligned}
$$

Using the Markov property of the Brownian motion, one readily justifies the equalities

$$
\begin{aligned}
& P\left[\sigma B_{1}=x^{\prime}, \sigma B_{\theta}=y \mid \sigma B_{0}=x\right] \\
& \quad=P\left[\sigma B_{1}=x^{\prime} \mid \sigma B_{\theta}=y\right] P\left[\sigma B_{\theta}=y \mid \sigma B_{0}=x\right] \\
& \quad=\rho_{f p}(x, y, \theta \beta) \rho_{f p}\left[y, x^{\prime} ;(1-\theta) \beta\right]
\end{aligned}
$$

and

$$
\begin{aligned}
\mathbb{E}\left[e^{-\beta \int_{0}^{1} V\left(\sigma B_{u}\right) d u} \mid \sigma B_{1}=x^{\prime}, \sigma B_{\theta}=y, \sigma B_{0}=x\right] \\
=\mathbb{E}\left[e^{-\beta \int_{0}^{\theta} V\left(\sigma B_{u}\right) d u} \mid \sigma B_{\theta}=y, \sigma B_{0}=x\right] \\
\quad \times \mathbb{E}\left[e^{-\beta \int_{\theta}^{1} V\left(\sigma B_{u}\right) d u} \mid \sigma B_{1}=x^{\prime}, \sigma B_{\theta}=y\right] .
\end{aligned}
$$

Performing the transformation of coordinates $u^{\prime}=u-\theta$ in the second factor of the right-hand side of Eq. (A4) and employing the invariance of the Brownian motion under time translation

$$
\begin{aligned}
& \left\{\sigma B_{u+\theta} \mid \sigma B_{\theta}=y, \sigma B_{1}=x^{\prime}, u \geqslant 0\right\} \\
& \quad \stackrel{d}{=}\left\{\sigma B_{u} \mid \sigma B_{0}=y, \sigma B_{1-\theta}=x^{\prime}, u \geqslant 0\right\},
\end{aligned}
$$

one obtains

$$
\begin{aligned}
\mathbb{E}\left[e^{-\beta \int_{0}^{1} V\left(\sigma B_{u}\right) d u} \mid \sigma B_{1}=x^{\prime}, \sigma B_{\theta}=y, \sigma B_{0}=x\right] \\
=\mathbb{E}\left[e^{-\beta \int_{0}^{\theta} V\left(\sigma B_{u}\right) d u} \mid \sigma B_{\theta}=y, \sigma B_{0}=x\right] \\
\quad \times \mathbb{E}\left[e^{-\beta \int_{0}^{1-\theta} V\left(\sigma B_{u}\right) d u} \mid \sigma B_{1-\theta}=x^{\prime}, \sigma B_{0}=y\right] .
\end{aligned}
$$

Let us focus on the term

$$
\mathbb{E}\left[e^{-\beta \int_{0}^{\theta} V\left(\sigma B_{u}\right) d u} \mid \sigma B_{\theta}=y, \sigma B_{0}=x\right] .
$$

Performing the substitution of variables $u^{\prime}=u / \theta$ and employing the scaling property of the Brownian motion

$$
\begin{aligned}
& \left\{\sigma B_{u \theta} \mid \sigma B_{0}=x, \sigma B_{\theta}=y, u \geqslant 0\right\} \\
& \quad \stackrel{d}{=}\left\{\sigma \theta^{1 / 2} B_{u} \mid \sigma \theta^{1 / 2} B_{0}=x, \sigma \theta^{1 / 2} B_{1}=y, u \geqslant 0\right\},
\end{aligned}
$$

one proves

$$
\begin{aligned}
\mathbb{E}\left[e^{-\beta \int_{0}^{\theta} V\left(\sigma B_{u}\right) d u} \mid \sigma B_{\theta}=y, \sigma B_{0}=x\right] \\
\quad=\mathbb{E}\left[e^{-\beta \theta \int_{0}^{1} V\left(\sigma \theta^{1 / 2} B_{u}\right) d u} \mid \sigma \theta^{1 / 2} B_{1}=y, \sigma \theta^{1 / 2} B_{0}=x\right] \\
=\rho(x, y ; \theta \beta) / \rho_{f p}(x, y ; \theta \beta) .
\end{aligned}
$$

In a similar fashion, one demonstrates that

$$
\begin{aligned}
\mathbb{E}\left[e^{-\beta \int_{0}^{1-\theta} V\left(\sigma B_{u}\right) d u} \mid \sigma B_{1-\theta}=x^{\prime}, \sigma B_{0}=y\right] \\
\quad=\rho\left[y, x^{\prime} ;(1-\theta) \beta\right] / \rho_{f p}\left[y, x^{\prime} ;(1-\theta) \beta\right] .
\end{aligned}
$$

We now combine Eqs. (A1)-(A3) and (A5)-(A7) to obtain

$$
\begin{aligned}
\int_{\Omega} d P[\bar{a}] X_{\infty}\left(x, x^{\prime}, \bar{a} ; \beta\right) O\left[x_{r}(\theta)+\sigma B_{\theta}^{0}(\bar{a})\right] \\
\quad=\int_{\mathbb{R}} \rho(x, y ; \theta \beta) \rho\left[y, x^{\prime} ;(1-\theta) \beta\right] O(y) d y .
\end{aligned}
$$

With the help of Eq. (A8) and by cyclic invariance,

$$
\begin{aligned}
\int_{\mathbb{R}} d x & \int_{\Omega} d P[\bar{a}] X_{\infty}(x, \bar{a} ; \beta) O\left[x+\sigma B_{\theta}^{0}(\bar{a})\right] \\
& =\int_{\mathbb{R}} d x \int_{\mathbb{R}} d y \rho(x, y ; \theta \beta) \rho[y, x ;(1-\theta) \beta] O(y) \\
& =\int_{\mathbb{R}} d y \rho(y, y ; \beta) O(y) \\
& =\int_{\mathbb{R}} d x \int_{\Omega} d P[\bar{a}] X_{\infty}(x, \bar{a} ; \beta) O(x) .
\end{aligned}
$$

Moreover, since the function $O(x)$ is arbitrary, the last identity also implies that the random variables $x$ and $x$ $+\sigma B_{\theta}^{0}(\bar{a})$ have identical distribution functions under the probability measure

$$
\frac{X_{\infty}(x, \bar{a} ; \beta) d x d P[\bar{a}]}{\int_{\mathbb{R}} d x \int_{\Omega} d P[\bar{a}] X_{\infty}(x, \bar{a} ; \beta)} .
$$

By setting $O(x)=1$ in Eq. (A8), one obtains the well-known product formula

$$
\begin{aligned}
\rho\left(x, x^{\prime} ; \beta\right) & =\int_{\Omega} d P[\bar{a}] X_{\infty}\left(x, x^{\prime}, \bar{a} ; \beta\right) \\
& =\int_{\mathbb{R}} \rho(x, y ; \theta \beta) \rho\left[y, x^{\prime} ;(1-\theta) \beta\right] d y,
\end{aligned}
$$

which is seen to be a consequence of some basic properties of the Brownian motion.

\footnotetext{
${ }^{1}$ Quantum Monte Carlo Methods in Physics and Chemistry, edited by M. P. Nightingale and C. J. Umrigar (Kluwer, Drodrecht, 1999).

${ }^{2}$ C. Predescu and J. D. Doll, J. Chem. Phys. 117, 7448 (2002).

${ }^{3}$ C. Predescu, J. D. Doll, and D. L. Freeman, Asymptotic Convergence of
} 
the Partial Averaging Technique, e-print: http://arXiv.org/abs/cond-mat/ 0301525

${ }^{4}$ C. Predescu, Reweighted Methods: Definition and Asymptotic Convergence, e-print: http://arXiv.org/abs/cond-mat/0302171

${ }^{5}$ J. D. Doll, R. D. Coalson, and D. L. Freeman, Phys. Rev. Lett. 55, 1 (1985); R. D. Coalson, D. L. Freeman, and J. D. Doll, J. Chem. Phys. 85, 4567 (1986).

${ }^{6}$ C. Predescu, D. Sabo, and J. D. Doll, Numerical Implementation of Some Reweighted Path Integral Techniques, e-print: http://arXiv.org/abs/condmat/0305436

${ }^{7}$ M. F. Herman, E. J. Bruskin, and B. J. Berne, J. Chem. Phys. 76, 5051 (1982).

${ }^{8}$ A. Giansanti and G. Jacucci, J. Chem. Phys. 89, 7454 (1988).

${ }^{9}$ P. A. Fernandes, A. P. Carvalho, and J. P. P. Ramalho, J. Chem. Phys. 103, 5720 (1995)

${ }^{10}$ J. Cao and B. J. Berne, J. Chem. Phys. 91, 6359 (1989).

${ }^{11}$ M. Kolar and S. F. O'Shea, J. Phys. A 29, 3471 (1996).

${ }^{12}$ W. Janke and T. Sauer, J. Chem. Phys. 107, 5821 (1997)

${ }^{13}$ D. M. Ceperley, Rev. Mod. Phys. 67, 279 (1995).

${ }^{14}$ K. R. Glaesemann and L. E. Fried, J. Chem. Phys. 116, 5951 (2002).

${ }^{15}$ C. Chakravarty, M. C. Gordillo, and D. M. Ceperley, J. Chem. Phys. 109, 2123 (1998).

${ }^{16}$ J. D. Doll and D. L. Freeman, J. Chem. Phys. 111, 7685 (1999).

${ }^{17}$ B. Simon, Functional Integration and Quantum Physics (Academic, London, 1979).
${ }^{18}$ R. Durrett, Probability: Theory and Examples, 2nd ed. (Duxbury, New York, 1996), pp. 430-431.

${ }^{19}$ S. Kwapien and W. A. Woyczynski, Random Series and Stochastic Integrals: Single and Multiple (Birkhäuser, Boston, 1992), Theorem 2.5.1.

${ }^{20}$ C. Predescu and J. D. Doll, Phys. Rev. E 67, 026124 (2003).

${ }^{21}$ H. P. McKean, Jr., Stochastic Integrals (Academic, New York, 1969).

${ }^{22}$ P. Sindzingre, D. M. Ceperley, and M. L. Klein, Phys. Rev. Lett. 67, 1871 (1991).

${ }^{23}$ J. P. Neirotti, D. L. Freeman, and J. D. Doll, Phys. Rev. E 62, 7445 (2000).

${ }^{24}$ N. Metropolis, A. W. Rosenbluth, M. N. Rosenbluth, A. M. Teller, and E. Teller, J. Chem. Phys. 21, 1087 (1953).

${ }^{25} \mathrm{M}$. Kalos and P. Whitlock, Monte Carlo Methods (Wiley-Interscience, New York, 1986).

${ }^{26}$ M. Matsumoto and T. Nishimura, ACM Trans. Model. Comput. Simul. 8, 3 (1998)

${ }^{27}$ M. Matsumoto and T. Nishimura, in Monte Carlo and Quasi-Monte Carlo Methods 1998 (Springer-Verlag, New York, 2000), pp. 56-69.

${ }^{28}$ http://www.math.keio.ac.jp/ matumoto/emt.html

${ }^{29}$ S. S. Shapiro and M. B. Wilk, Biometrika 52, 591 (1965).

${ }^{30}$ W. H. Press, S. A. Teukolsky, W. T. Vetterling, and B. P. Flannery, $\mathrm{Nu}-$ merical Recipes (Cambridge University, Cambridge, 1992), Chap. 14.3.

${ }^{31}$ J. P. Valleau and S. G. Whittington, in Statistical Mechanics, edited by B. J. Berne (Plenum, New York, 1977), Chap. 4, p. 145. 
The Journal of Chemical Physics is copyrighted by the American Institute of Physics (AIP). Redistribution of journal material is subject to the AIP online journal license and/or AIP copyright. For more information, see http:/ojps.aip.org/jcpo/jcpcr/jsp Copyright of Journal of Chemical Physics is the property of American Institute of Physics and its content may not be copied or emailed to multiple sites or posted to a listserv without the copyright holder's express written permission. However, users may print, download, or email articles for individual use. 
The Journal of Chemical Physics is copyrighted by the American Institute of Physics (AIP). Redistribution of journal material is subject to the AIP online journal license and/or AIP copyright. For more information, see http://ojps.aip.org/jcpo/jcper/jsp 